\title{
Las enfermedades respiratorias del adulto mayor en Chile: un desafío a corto plazo
}

\author{
RICARDO SEPÚLVEDA M.*
}

\section{Respiratory diseases of older adults in Chile: a short-term challenge}

The older population is increasing very rapidly in Chile; every 20 years this population is growing twice. Chronic Non Communicable Disease (CNCD) will be the main health problem, not only in developed countries released World Health Organization. Preventive actions such as annual influenza vaccination, anti-pneumococcal immunization, avoidance of cigarette smoke and air pollution and swallowing disorder early recognition have proven to reduce the risk of developing low respiratory infections. The management of Asthma and COPD (frequents CNCD) in elderly populations will require a growing number of well-trained health personnel. Despite a reduction of all age cases of Community Acquired Pneumonia (CAP) in Chile, there are recognized risk factors that make the elderly population fragile. Quality standards application in patients under respiratory care have been shown to have a positive impact not only in sanitary health indicators, but in patients quality of life. This is a challenge to face right now.

Key words: Aged; respiratory tract infections; pneumonia; asthma; pulmonary disease, chronic obstructive; risk factors; quality of life.

\section{Resumen}

Chile sufre un envejecimiento acelerado de su población, por lo que la cantidad de adultos mayores se ha duplicado en los últimos veinte años. Las enfermedades crónicas no trasmisibles, entre ellas el asma bronquial y EPOC, como lo ha señalado la Organización Mundial de la Salud, aumentarán su peso sanitario relativo y por ende requerirán de equipos médicos capacitados en su manejo. Al mismo tiempo, en nuestro país se aprecia una reducción de la tasa de neumonías incluyendo a los grupos etarios más avanzados. Existen diferentes factores favorecedores de las infecciones respiratorias bajas que deben ser considerados en la prevención de estos cuadros; sobresaliendo los trastornos de la deglución, las rinopatías crónicas y factores asociados a la inmunosenescencia. Se enfatiza el valor de la inmunoprevención en esta edad y la necesidad de definir estándares de calidad para lograr impacto sanitario y mejoría de la calidad de vida de nuestros adultos mayores.

Palabras clave: Adulto mayor; enfermedades del tracto respiratorio; neumoní; asma; EPOC: factores de riesgo; calidad de vida.

\section{Introducción}

La distribución demográfica de la población chilena está cambiando en forma acelerada tal como lo ha demostrado en forma preliminar el censo nacional de 2017. En él, se observa un aumento progresivo del grupo de personas mayores tal como sucede en la mayoría de las sociedades desarrolladas. El grupo mayor de 65 años ha llegado a constituir el $11,4 \%$ de los 17.574 .014 habitantes censados, lo que representa practicamente el doble de los censados el año 1992 ${ }^{1}$. El índice de envejecimiento (relación porcentual entre sujetos de 65 años y más y los menores de 15 años) ha aumentado de 22,3\% el año 1992 hasta $56,8 \%$ en el último censo.

* Profesor titular, Facultad de Medicina, Universidad de Chile.

Coordinador de la Comisión de Asma en el Adulto, Sociedad Chilena de Enfermedades Respiratorias. 
La Segunda Encuesta Nacional de Calidad de Vida en la Vejez 2010 (SENAMA-UC) mostró que el $25 \%$ de los chilenos mayores de 65 años encuestados señalaban que en el último año habian presentado tos persistente o ahogos, $12,3 \%$ padecian de EPOC y $11 \%$ sufría de asma bronquial.

Las enfermedades respiratorias (ER) están consideradas a nivel mundial, entre las principales causas de discapacidad severa de los adultos mayores (AM), superando incluso a las producidas por los accidentes cerebrovasculares. Esto no es diferente en nuestro pais, donde las ER poseen además un considerable peso sanitario en este grupo etario. De las hospitalizaciones totales de 2002 el grupo mayor de 65 años constituía el $16,7 \%$. Al considerar sólo las hospitalizciones por causa respiratoria (J00-J90) ya ese año, el grupo de adultos mayores mostraba un exeso comparativo del 40\%. El año 2014 se mantenia aproximadamente el mismo número de hospitalizaciones totales en el pais, pero la proporción de AM hospitalizados por cualquier causa se había elevado a un $20 \%$. La proporción de ese grupo hospitalizado por causas respiratorias se elevó hasta un 30\% del total.

Entre los años 2000 y 2010 se ha evidenciado a nivel nacional un aumento de la tasa de mortalidad de los mayores de 65 años, atribuida a las enfermedades bronquiales obstructivas (CIE 10 J40-J47), siendo este incremento aún más significativo en el grupo femenino que casi triplicó la tasa de los hombres por estas mismas causas ${ }^{2}$. Situación inversa ha sucedido con los fallecimientos por neumonía, que han disminuído su tasa en este período desde 324/100.000 habitantes el año 2000 hasta 222/100.000 el 2010. Es decir, en nuestro pais el impacto de las enfermedades respiratorias depende fundamentalmente de las enfermedades crónicas, con una disminución significativa del impacto de las infecciones.

En general al atender a los adultos mayores, se tiende a considerar principalmente su edad cronológica sin evaluar sus alteraciones funcionales, ni sus comorbilidades y menos aún sus propios deseos. Posiblemente por ello la comunidad médica conserva aún una actitud pesimista sobre el pronóstico de la mayoría de los padecimientos de los ancianos. En un estudio efectuado en pacientes portadores de EPOC hospitalizados en Unidades de Terapia Intensiva del Reino Unido $^{3}$, se demostró la existencia de una gran discrepancia entre el pronóstico de sobrevida a seis meses estimada por los médicos y la acaecida en la realidad durante su seguimiento. Se evidenció en esta observación, que la edad como factor aisla- do, poseía poca significación entre las diferentes variables que podían determinar la sobrevida de estos pacientes después de su estadía en la UTI.

Una proporción importante de las enfermedades respiratorias de los adultos mayores se asocia a los riesgos inhalatorios a los que los individuos han estado expuestos durante su vida, sobresaliendo entre ellos el hábito tabáquico, la contaminación ambiental, las infecciones respiratorias durante la infancia y los contaminantes de origen laboral. Estas condiciones, muy frecuentes en nuestras comunidades, asociadas a las comorbilidades tan propias de este grupo etario, pueden explicar la importante carga sanitaria que causa la tercera edad ${ }^{4}$. Debe considerarse también en las enfermedades de este grupo, el deterioro fisiológico del aparato respiratorio, la existencia de una alta frecuencia de síntomas respiratorios crónicos en la población general y el aumento proporcional de la mortalidad respiratoria que se observa en los individuos de edad avanzada en comparación con los jóvenes ${ }^{5}$. Las razones mencionadas hacen necesario conocer el comportamiento de las enfermedades respiratorias en el adulto mayor, sus características clínicas y los requerimientos sanitarios que ellos determinan y así evitar errores clínicos, que se producen al utilizar datos obtenidos de poblaciones jóvenes, extrapolándolos a las personas de la tercera edad ${ }^{6}$.

Debe agregarse a la multimorbilidad asociada a la edad, la existencia de una condición propia de la edad avanzada no frecuentemente evaluada, la inmunosenesencia que dificulta aún más el manejo de las enfermedades respiratorias en el grupo geriátrico y que ha obligado a investigar para ellos, estrategias diferentes para el manejo de las enfermedades inmunoprevenibles ${ }^{7}$. Durante el envejecimiento se modifican tanto la inmunidad innata como la adquirida, contribuyendo a explicar, a lo menos parcialmente, la mayor frecuencia de enfermedades infecciosas, autoinmunitarias y tumorales tan propias de esta etapa de la vida.

El envejecimiento acelerado sufrido en los últimos años por la población, ha determinado un desplazamiento de las afecciones respiratorias infecciosas por las enfermedades crónicas no transmisibles, influyendo en el deterioro de la calidad de vida, la carga sanitaria y en las cifras de mortalidad de nuestro país. En la Figura 1 se compara la mortalidad por neumonía y enfermedades respiratorias crónicas en el último decenio).

En esta publicación, haremos un análisis de los factores que influyen en las enfermedades respiratorias de la tercera edad, las politicas sanitarias implementadas y su evolución en el último decenio. 


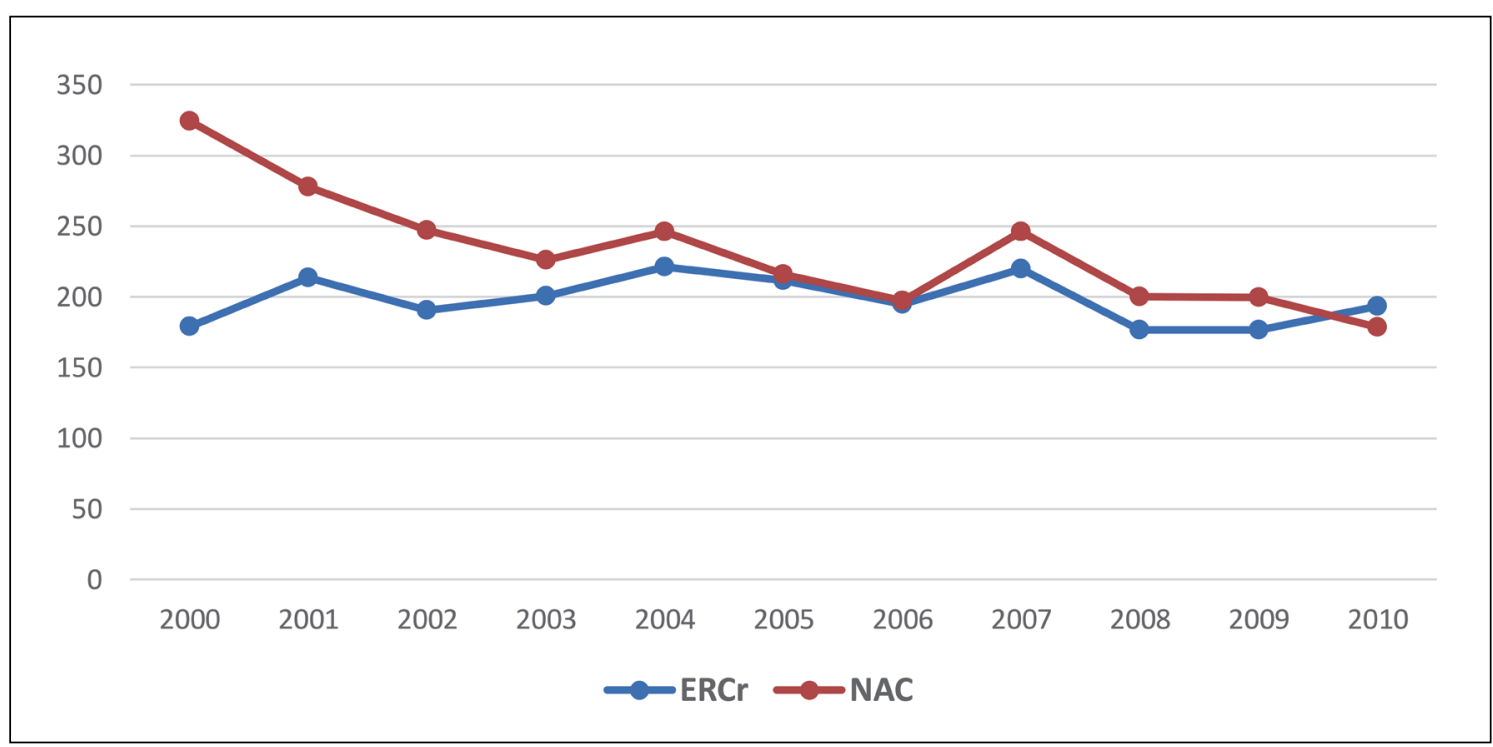

Figura 1. Comparación de la mortalidad por neumonía y enfermedades respiratorias crónicas (ERCr) en el período 2000-2010. Departamento de Estadísticas e Información de Salud (NAC). Ministerio de Salud, Chile.

El envejecimiento se acompaña de un aumento de la rigidez de la caja torácica, pérdida de la fuerza de retracción elástica pulmonar y disminución de la fuerza de los músculos respiratorios. Ello determina una reducción tanto de los flujos como de los volúmenes pulmonares, un aumento del volumen residual y de la ventilación frente al ejercicio ${ }^{8}$. Si a esto se agrega la frecuente aparición de disnea en esta edad asociada a la pérdida de masa muscular, sedentarismo, alteraciones cardiovasculares subclínicas, alteraciones metabólicas, anemia, entre otras; se hace aún más dificil la interpretación de los valores espirométricos en estos pacientes. Por ello no es infrecuente que se catalogue como anormal un estudio funcional respiratorio de un adulto mayor sintomático si se le compara con una tabla de referencia construida en base a una población que no incluye un número representativo de sujetos de su grupo etario. De este modo puede atribuírsele al sistema respiratorio las limitaciones del paciente, sin buscar su causalidad en alteraciones de otros órganos o sistemas que pudieran ser tratadas en forma eficiente al ser identificada su responsabilidad causal.

La nariz en el adulto mayor presenta alteraciones fisiológicas y estructurales; debilitamiento del cartílago nasal, atrofia de su mucosa y modificación de sus secreciones, por lo que es frecuente la aparición de obstrucción nasal, prurito, descarga posterior, anosmia, rinorrea e infecciones repetidas $^{9}$. Estas alteraciones suelen determinar un deterioro de la calidad de vida que incluso puede ser más significativo que la obstrucción bronquial. Con frecuencia se asocian estos síntomas con una posible causa alérgica y por ende se tiende a prescribir con innecesaria frecuencia fármacos antihistaminicos o corticoides tópicos sin considerar sus potenciales efectos colaterales sobre todo en este grupo etario. La rinitis crónica de la tercera edad puede contribuir al desarrollo de respiración bucal, anosmia y ageusia que suelen ser factores adyuvantes de los trastornos alimentarios frecuentes a esta edad. También la descarga posterior puede asociarse con bronquitis aspirativas secundarias e incluso se ha demostrado que la persistencia de esta alteración impide el control adecuado del asma bronquial. En un grupo de adutos mayores de 65 años que residían en 21 centros de larga estadía ${ }^{10}$, el $22 \%$ declaraba poseer síntomas de rinosinusopatía crónica. Es decir, los trastornos rinosinusales de esta población son frecuentes y deben ser especialmente considerados si se quiere lograr resolver los problemas respiratorios que alteran la calidad de vida de esta población.

La deglución es un complejo mecanismo neuronal que requiere para su buen funcionamiento una eficiente interacción entre la via respiratoria y la digestiva. Necesita coordinar impulsos sensitivos, motores y nocioceptivos, tanto de la orofarinx como de la laringe utilizando para esto los nervios craneales V, VII, IX, X, como vía aferente hacia el SNC, lugar donde interactúan con otros impulsos provenientes de la corteza cerebral. Alteraciones de este circuito, que suele deteriorarse al avanzar los años o como secuelas 
de enfermedades cerebrovasculares, determinan que la disfagia sea también un sintoma frecuente a esta edad, y en pacientes que han padecido accidente vascular encefàlico, enfermedad de Parkinson, enfermedad de Alzheimer e incluso en los mayores de 70 años sin esas patologías (presbifagia). Esto se ha demostrado en repetidas oportunidades en residentes de hogares de ancianos ${ }^{11}$. En general, esta alteración de la deglución es subestimada, a pesar de ser responsable de problemas respiratorios y nutricionales significativos en este grupo etario.

Los trastornos de la deglución contribuyen al desarrollo de la neumonía aspirativa, complicación severa y frecuente en los gerontes cuando no es identificada oportunamente y no se toman las medidas preventivas requeridas. Este trastorno funcional, debe ser investigado dirigidamente cada vez que se atiende a un paciente mayor y se pretende prevenir las importantes complicaciones asociadas a la aspiración de alimentos o secreciones de la cavidad oral. La observación directa de la deglución ininterrumpida de un vaso de agua, es un método sencillo y eficiente para evaluar esta alteración cuando ella se sospecha. La aparición coincidente de tos o de un trastorno de la fonación posterior debe ser considerado confirmatorio de la sospecha clínica ${ }^{12}$.

La neumonía aspirativa, es un cuadro de alta ocurrencia en este grupo etario, y si bién no se dispone de datos de su incidencia poblacional, se ha demostrado que ella se asocia con el envejecimiento y con la presencia de enfermedades subyacentes. En residentes de hogares de ancianos portadores de trastornos de la deglución, su frecuencia puede llegar al 50\% en el primer año de estadía, con una mortalidad sobre el $45 \%{ }^{13}$. En un estudio consecutivo de pacientes mayores de 70 años hospitalizados por neumonía en una unidad geriátrica, el $55 \%$ de ellos presentaba signos clínicos de disfagia orofaríngea. Se concentraba en los de edad más avanzada, y ellos poseían comparativamente una menor capacidad funcional, mostraban peor estado nutricional y poseían un índice de Fine de severidad de la neumonía más alto. Su mortalidad era significativamente más elevada a los 30 días y al año de seguimiento después de ocurrida la neumonía ${ }^{14}$. Así, la disfunción orofaríngea es muy frecuente en adultos mayores y es un factor de gravedad en la neumonía de este grupo etario.

De un modo similar, alteraciones de los mecanismos de defensa, tales como abolición de la tos, la reducción de la limpieza faríngea, el aumento de la colonización orofaringea y la debilidad del sistema inmunitario muchas veces asociado a la desnutrición y comorbilidades, contribuyen al aumento de la frecuencia de las neumonias en este grupo etario, sobre todo en quienes poseen riesgo tabáquico y cavidad oral en malas condiciones.

Los principales microoorganismos aislados en las neumonías aspirativas adquiridas en la comunidad son el Streptococcus pneumoniae, Staphylococcus aureus, Haemophilus influenzae y se agregan los bacilos gramnegativos en las neumonias nosocomiales ${ }^{15}$. El S. pneumoniae, continúa siendo uno de los principales agentes patógenos en el grupo de pacientes de edad avanzada y en los portadores de condiciones clínicas asociadas a déficit inmunitarios que requieren hospitalización. Esta bacteria es responsable de un número considerable de fallecimientos en nuestro país tal como en el resto del mundo ${ }^{16}$. Por ello las infecciones neumocócicas han sido identificadas como un problema importante de salud pública y varias organizaciones internacionales han planteado la utilización de la vacuna antineumocócica en los adultos pertenecientes a esos grupos de riesgo. Actualmente existen dos vacunas antineumocócicas, la vacuna conjugada ( 7,10 y 13 valentes) de uso preferencial en niños, y la vacuna polisacárida 23 valente para ser usada en grupos de riesgo y adultos mayores. Se estima que esta última es capaz de conferir una protección de 50 a $60 \%$ durante cinco años a estos dos grupos.

$\mathrm{Si}$ las infecciones respiratorias bajas son importantes en el adulto mayor, tanto más son las enfermedades respiratorias crónicas, sobresaliendo por su frecuencia el asma bronquial y la EPOC.

¿Se aplican las guías de práctica clínica en forma similar en este grupo etario a como se hace en el adulto joven? ¿Qué limitaciones tenemos en la práctica clínica para diferenciarlas? Las Guías GOLD 2017 fueron explícitas en señalar que el diagnóstico de EPOC se basa en la "existencia de síntomas respiratorios y obstrucción bronquial persistente debidas a alteraciones bronquiales y/o alveolares generalmente causadas por una exposición significativa a partículas o gases dañinos". Los síntomas respiratorios son frecuentes en el adulto mayor por múltiples causas, y la demostración de obstrucción bronquial requiere de la aplicación de una espirometría de limitada utilización en nuestro medio más aún en los sujetos mayores. Esto explica el elevado subdiagnóstico de esta frecuente condición. A lo largo de nuestro país poseemos en la mayoría de los Centros de Salud Familiar, la amplia disponibilidad de espirómetros, sin embargo, aún su utilización es limitada. No es posible confirmar el diagnóstico 
de EPOC y menos aún hacer diagnóstico diferencial con asma en el adulto mayor sin disponer de los valores espirométricos. Este es un estándar mínimo de calidad en el diagnóstico de la EPOC.

El asma bronquial con frecuencia ha sido considerada una enfermedad de individuos jóvenes. Sin embargo, las consultas por crisis bronquiales obstructivas reversibles en los mayores de 65 años son de alta frecuencia, y las hospitalizaciones con este diagnóstico son más frecuentes que en los menores de esa edad. La afirmación que la "EPOC es enfermedad de individuos mayores y el asma sólo de los jóvenes", es otro de los mitos que ha impedido a muchos adultos mayores gozar de una mejor calidad de vida al lograr un control adecuado de su asma bronquial.

\section{Bibliografía}

1.- RESULTADOS DEFINITIVOS DEL CENSO 2017. Instituto Nacional de Estadísticas. Disponible en: www. ine.cl

2.- WILDMAN MJ, SANDERSON C, GROVES J, REEVES BC, AYRES J, HARRISON D, et al. Implications of prognostic pessimism in patients with chronic obstructive pulmonary disease (COPD) or asthma admitted to intensive care in the UK within the COPD and asthma outcome study (CAOS): multicentre observational cohort study. Brit Med J 2007; 335: 1132.

3.- ESTADÍSTICAS DE MORTALIDAD EN ADULTOS MAYORES DE 65 AÑOS 2010. Departamento de Estadísticas e Información de Salud, Ministerio de Salud, Chile. Disponible en: www.minsal.cl

4.- MALlET L, SPINEWINE A, HUANG A. The challenge of managing drug interactions in elderly people. Lancet 2007; 370: 185-91.

5.- MURRAY CJ, LÓPEZ AD. Evidence-based health policy-lessons from the Global Burden of Disease Study. Science 1996; 274: 740-3.
6.- PELLEGRINO R ${ }^{1}$, VIEGI G, BRUSASCO V, CRAPO RO, BURGOS F, CASABURI R, et al. Interpretative strategies for lung function test. Eur Respir J 2005; 26 : 948-68.

7.- DEL GIUDICE G, GORONZY JJ, GRUBECKLOEBENSTEIN B, LAMBERT PH, MRKVAN T, STODDARD JJ, et al. Fighting against a protean enemy: immunosenescence, vaccines, and healthy aging. NPJ Aging Mech Dis 2017; 4: 1.

8.- STANOJEVIC S, WADE A, STOCKS J. Reference values for lung function: past, present and future. Eur Resp J 2010; 36: 12-9.

9.- PINTO JM, JESWANI S. Rhinitis in the geriatric population. Allergy Asthma Clin Immunol 2010; 6 (1): 10.

10.- MENDES A, PAPOILA AL, CARREIRO-MARTINS P, BONASSI S, CAIRES I, PALMEIRO T, et al. The impact of indoor air quality and contaminants on respiratory health of older people living in long-term care residences in Porto. Age Ageing 2016; 45: 136-42.

11.- CLAVÉ P, VERDAGUER A, ARREOLA V. Oralpharyngeal dysphagia in the elderly. Med Clin (Barc) 2005; 124: 742-8.

12.- DEPIPPO KL, HOLAS MA, REDING MJ. Validation of 3-oz water swalow test for aspiration following stroke. Arch Neurol 1992; 49: 1259-61.

13.- COOK IJ, KAHRILAS PJ. AGA technical review on management of oropharyngeal dysphagia. Gastroenterology 1999; 116: 455-78.

14.- CABRE M, SERRA-PRAT M, PALOMERA E, ALMIRALL J, PALLARES R, CLAVÉ P. Prevalence and prognostic implicacións of dysphagia in elderly patients with pneumonia. Age Ageing 2010; 39: 39-45.

15.- ALMIRALL J, CABRÉ M, CLAVÉ P. Aspiration pneumonia. Med Clin (Barc) 2007; 129: 424-32.

16.- 23-valent pneumococcal polysaccharide vaccine. WHO position paper. Wkly Epidemiol Rec 2008; 83: 373-84.

17.- NICE guideline. Asthma: diagnosis, monitoring and chronic asthma manegment. Published 29 November 2017. Disponible en: www.nice.org.uk/guidance/ng80.
Correspondencia a:

Dr. Ricardo Sepúlveda M.

Santa Magdalena 75 Oficina 701,

Comuna de Providencia, Santiago, Chile.

Email: ricardolsepulveda2511@gmail.com 